\title{
Factors Affecting Latino Adults' Use of Antibiotics for Self-Medication
}

\author{
Arch G. Mainous, III, PhD, Vanessa A. Diaz, MD, MS, and Mark Carnemolla, BA
}

Background: Recent data has suggested the use of antibiotics acquired without a prescription by Latinos in the United States. We explored Latino adults' experience in treating common infections particularly in regards to self-medication with antibiotics.

Methods: We conducted 3 focus groups $(n=28)$ in Charleston, South Carolina, with Latino adults ( $\geq 18$ years of age) recruited throughout the community: 12 women and 16 men, ranging in age from 18 to 52 years. All of the participants were immigrants, $89 \%$ noted Mexico as their country of origin. Focus groups were conducted in Spanish and audiotaped. Transcripts were translated into English and then translated back to Spanish to assure consistency of the language. Themes were identified using an editing style.

Results: Participants' previous experiences in countries with limited restrictions on antibiotics influenced acquisition of antibiotics without a prescription in the United States. Participants believed that physician visits for a diagnosis and prescription were unnecessary when the patient was familiar with the symptom and it had previously responded to antibiotic treatment. Access to care was not reported to be a significant barrier to a physician visit when individuals felt they were "sick" or children were the patients. Participants reported using local tiendas (small stores in Latino neighborhoods that sell ethnically consistent and imported products) and importation of medication to meet their need for selfmedication with antibiotics. The role of self-medication in the development of antibiotic resistance was essentially unknown among the participants.

Conclusions: Successful interventions to improve use of antibiotics need to be culturally sensitive to specific attitudes and behaviors found in the Latino population. ( $\mathrm{J}$ Am Board Fam Med 2008;21:

128-134.)

Antibiotics serve a very useful therapeutic purpose in eradicating pathogens. Unfortunately, excessive use of antibiotics has contributed to the spread of the important clinical and public health problem of antibiotics resistance. ${ }^{1,2}$ In some countries, the use of antimicrobials without a prescription is encouraged by the lack of laws restricting antibiotic sales or a failure to enforce those laws. ${ }^{3-6}$ These issues are particularly problematic in Latin America., ${ }^{6,7}$

Health beliefs and practices are integrated into one's ethnic and cultural orientation. ${ }^{8-10}$ Some be-

This article was externally peer reviewed.

Submitted 8 June 2007; revised 20 August 2007; accepted 24 August 2007.

From the Department of Family Medicine, Medical University of South Carolina, Charleston.

Funding: This work is funded in part by the Blue CrossBlue Shield Foundation of South Carolina, the Robert Wood Johnson Foundation, and the National Institute on Aging (1 P30 AG21677).

Conflict of interest: none declared.

Corresponding author: Arch G. Mainous, III, PhD, Department of Family Medicine, Medical University of South Carolina, 295 Calhoun Street, Charleston, SC 29425 (Email: mainouag@musc.edu). lief systems that have roots in Latin American cultures may encourage the overuse of antibiotics, particularly the use of nonprescribed antibiotics. Latinos are more likely than non-Latino whites to believe that antibiotics are necessary for their child's or their illness. ${ }^{11,12}$ That antimicrobials should be available without a prescription is a prevalent attitude among Latinos. ${ }^{13,14}$ Recent evidence in the Latino community in South Carolina indicates a high level of importation of antibiotics into, and the acquisition of antibiotics without a prescription in, the United States. ${ }^{14}$ Moreover, many respondents suggested self-medicating with antibiotics was preferable to going to the doctor.

The Latino population has historically been highly concentrated in certain metropolitan areas, such as New York and Los Angeles, and the southwestern United States. More recently however, this population has dispersed to states with smaller cities or more rural areas, such as South Carolina. The Hispanic population in South Carolina increased $211 \%$ from 1990 to 2000, making it the state with the fifth largest Latino growth during 
this period. ${ }^{15}$ However, because Hispanics overall make up only $2.0 \%$ of the population in this state, Latinos in South Carolina may have different strategies when seeking care and managing illness than individuals in more urbanized areas with a higher concentration of Latino populations. This focus group study elicited Latino adults' experiences in treating common infections and acquiring treatments while capturing their reasons for using antibiotics as treatment.

\section{Methods}

\section{Participants}

This study received Institutional Review Board approval from the Medical University of South Carolina. Self-identified Latinos adults ( $\geq 18$ years of age) were recruited through flyers placed at clinical sites, community centers and stores in Charleston, South Carolina. Individuals were volunteers and were not selected based on current illness. We conducted 3 focus groups with adult members of the Latino community to assess factors that may contribute to inappropriate use of antibiotics.

Twenty-eight individuals (12 women and 16 men) with an age range from 18 to 52 years (mean age, 29 years) participated in one of 3 focus groups. Group attendance ranged from 5 to 12 participants. All of the participants were immigrants, with the vast majority (89\%) having Mexico as their country of origin; participants also came from Guatemala $(\mathrm{n}=2)$ and Honduras $(\mathrm{n}=1)$. All but 2 of the participants $(7 \%)$ were at least 15 years old when they arrived in the United States. All of the participants spoke Spanish as their native language. The participants had attained limited formal education; 15 had less than a high school degree and only one had more education than high school. Many of the participants were familiar with the US health care system, and 19 of $28(68 \%)$ had visited a physician in the United States.

\section{Data Collection}

Data were collected over 3 months, between October and December 2006. Focus groups were conducted by a trained bilingual, Latino investigator and a bilingual assistant. All groups were conducted in Spanish, per participant preference. Demographic information was obtained via a survey. The investigator had a list of general and probing questions for pre-planned topics of discussion. These questions were adjusted for later groups based on trends and patterns requiring further elucidation. General topic questions used in the focus groups have been translated into English and are presented in Appendix 1. These general topic questions were asked in the context of the ongoing discussion. The group discussions were audiotaped and transcribed in Spanish, then translated into English and backtranslated to ensure accuracy. The investigators also took field notes and participated in debriefings after the discussions.

\section{Analysis}

Transcripts were analyzed in a systematic manner by 3 independent investigators, 2 of whom were bilingual and 1 of whom was ethnicity concordant and spoke Spanish as her native language. To reduce the influence of preconceptions about the data, a commonly used interpretive form of analysis known as editing was used. ${ }^{16}$ This interpretive form of analysis involves searching for meaningful segments of text and organizing them into categories and themes. The aim of analysis was to find commonly recurring themes, trends, and patterns within group discussions relating to self-medication with antibiotics and the acquisition of antibiotics without a prescription. Words used, context, internal consistency, specificity of responses, and overlying themes were considered. Tone and nonverbal communication was assessed through the field notes. Saturation, which was determined by the redundancy of data and respondent validation of themes, occurred after 3 focus groups. Data collection was terminated once saturation was reached. $N^{*}$ VIVO software (QSR International, Cambridge, Mass.) was used to facilitate data abstraction and analysis. Members of the team reviewed the transcripts individually and then met on several occasions to compare segments of text from the transcribed interviews, explore the themes that emerged in the discussion, and reach consensus about interpretation of the text and themes.

\section{Results}

The investigators identified several themes emerging from these focus group discussions: the role of previous experience affecting patterns of self-medication with antibiotics; the need to see a physician for a simple illness to receive antibiotics; the utility of physician visits for the diagnosis and treatment 
of children; informal strategies to obtain antibiotics without a prescription; and the risks of self-medicating with antibiotics. An additional point of interest was the suggestion by the participants regarding ways to encourage appropriate use of antibiotics and less self-medication with antibiotics obtained without a prescription. These are discussed in more detail below, with illustrative quotes from participants.

\section{Self-Medication with Antibiotics is Driven by Previous Experience with Antibiotics}

The first theme to emerge was related to participants' previous success with antibiotics. When they get symptoms that they have experienced before, they seek the treatment that seemed successful during prior episodes of those symptoms. The participants reported that for the first episode of a specific illness they had usually sought medical care and a diagnosis from a health care worker, many times a pharmacist. They felt comfortable with a self-diagnosis if they had a subsequent episode with a similar symptom complex. Thus, self-medication seems appropriate because they are relying on previous experience with similar symptoms to identify the condition, diagnosis, and treatment. This search for previously used treatments even extended to the desire to self-medicate with injectable antibiotics gained without a prescription, a behavior with substantial public health implications.

- "I also think that sometimes when you get sick and don't know what medication to take, you go to the doctor and get some medication. You see this medication worked for you, and there were no side effects. Then next time you have the same problem you don't go to the doctor. Right? You don't go the doctor. You return to the pharmacy. There are medications that are controlled, that are strong. Like narcotics, they are controlled, they won't sell them to you. But when it's an antibiotic, they will sell it to you."

- "Over in Mexico, usually when you go to the pharmacy there is always someone who knows about medicine, similar to a doctor. You go and consult with them, and they tell you for what you have, use this."

- "When you have a flu, the flu, you have symptoms... Then you go to the pharmacy and ask for some kind of antibiotic, usually penicillin, or injectable penicillin. You can buy that at the pharmacy."

\section{Barriers for Treating a Simple Illness: a Physician Visit is Not Worth Dealing With}

The next theme to emerge was one of ambivalence toward a physician consultation for the treatment for common infections. Cost of care and language barriers were weighed against the benefits of a physician visit for these seemingly uncomplicated illnesses. The participants noted that they would seek a physician consultation in an emergency or for something serious. However, because they knew what needed to be done for this condition, the extra cost and language barriers seemed excessive. Moreover, when some participants had some concerns about the veracity of their self-diagnosis they called health care providers in Mexico to provide an expert opinion. This was still seen as easier and less expensive than seeing a physician in the United States.

- "If I already know my symptoms and what medicine heals me, I'm not going to go to the doctor so they can give me a prescription there. If I go, they'll charge me just for the visit, and then I have to go buy the prescription and that's more money, another cost. If I know what medication I'm going to take and will work for me, I'll go buy it and it's cheaper for me."

- "I've already been sick like that. Why should I go to the doctor if I know [the sickness] goes away with that [medication]. If it doesn't go away with that [medication], then I'll go to the doctor."

- "And then he [a doctor in Mexico] tells me what to take. He tells me, you should take this, and that has worked well for me. He tells me, you should not take this other thing, you have to eat right, and like that gives me all the indications over the phone. This works for me since like the other gentleman said, then I don't have to go to the doctor here."

\section{A Physician Visit is Necessary for the Diagnosis and Treatment for Children}

As opposed to the self-management strategies that the participants would use for themselves, they felt that it was important to consult with a physician for diagnosis and treatment of children. Adults have previously been diagnosed and treated for this recognizable symptom complex so they feel comfort- 
able in bypassing a physician visit before obtaining treatment. Children, on the other hand, cannot verbalize well and have no frame of reference or experience in which to place this current illness, thereby necessitating the need for physician diagnosis.

- "When you are sick yourself with a flu, you sometimes just wait it out. But if it is a child who is sick, you must take them to the doctor. With children you don't wait."

- "With kids you are more careful because they are small and don't know what is hurting them. In contrast an adult already knows what one has and what hurts and that sort of thing, but a child doesn't."

- "Children must go to the doctor. Because antibiotics are too strong. Children are weaker and can't take as much."

\section{Informal Strategies Used to Facilitate Access to Antibiotics}

The participants noted a variety of strategies that they used to obtain antibiotics instead of the usual US system of obtaining a prescription from a health care provider. These included having relatives acquire antibiotics without a prescription in Mexico and then mail them to the participants, bring antibiotics into the United States with them and use local tiendas (small stores in Latino neighborhoods that sell ethnically consistent and imported products) to acquire antibiotics without a prescription. The respondents seemed to recognize that having the tiendas sell antibiotics without a prescription was against US law, but because the practice was consistent with Mexican culture they did not seem troubled by this behavior. These strategies were seen as ways of bypassing what the participants considered to be an expensive and excessively regulated system in an effort to obtain treatment for common problems.

- "Mexican tiendas. I have heard a lot of people buy medications that if you go to the American pharmacies you have to have a prescription, but at Mexican tiendas you can buy them without a prescription.”

- "I ask them to get me the medicine in Mexico, since for $\$ 100$ they send me many things. That's the same as a doctor's visit. I ask my mother to send me medicine from Mexico, what we use most often."

\section{People Realize There are Some Personal Risks to Antibiotic Use but no Public Health Implications}

The participants voiced an understanding of the acute risk with the use of antibiotics, specifically allergic reactions that could lead to rashes and even death. However, there was little to no awareness of the concept of antibiotic resistance. One participant who had some health care training could explain the concept of antibiotic resistance and another participant talked about the body growing tolerant to antibiotics. These attitudes and knowledge seem to drive the desire for an initial diagnosis and concern about the first use of antibiotics for that condition, but fear or concern regarding repeat antibiotic use was greatly diminished.

- "I have seen at times where a person says, if 500 $[\mathrm{mg}]$ is good for me then I'll take 1000 [mg]. They get full of hives, I don't know, like an allergy. Imagine if a person is also sick with let's say a heart problem or suffers from blood pressure. It can be a pretty serious problem. People can get worse instead of getting better."

- "Well, there are also a lot of news reports about different antibiotics that can cause reactions in the brain, respiratory arrest. Any type of reaction can happen, if you don't know how to use them."

\section{Encouragement to See a Doctor Before Using Antibiotics Viewed as a Positive Strategy to Decrease Self-Medication}

The group participants commonly voiced a suggestion that the best message to the Latino community regarding antibiotics is that they need to go to the doctor to get a consultation on the illness rather than treating themselves. However, that message is constrained by the real-life barriers and constraints of this population's ability to receive health care. Furthermore, this proposed strategy is intertwined with the belief that a doctor visit is unnecessary and simply a mechanism to financially enrich the doctor. The message that the participants suggested might be the most effective was to encourage a doctor visit because of the risk of drug complications and allergic reactions when self-medicating.

- "The doctor gives you the prescription but when you go to the pharmacy they will not give you 
anything because you need an ID, driver's license. That is the problem. That is why one self-prescribes."

- "The doctor gave me syrup but he wanted to see me to charge me more. He knows what I need but wants to charge me for an unnecessary visit knowing I just need a prescription.”

- "There are people that have allergies that the medicine agrees with them and others that the medicine does not. If one takes an antibiotic they do not know that one can get sick, gravely ill.”

The results suggest the interplay between previous experience, barriers to care experienced by this population, and the complexity in crafting potential interventions to address this behavior and encourage appropriate antibiotic use.

\section{Discussion}

The Latino adults discussing their use of antibiotics in this study showed a preference for self-medication of common infections with antibiotics obtained without a prescription. Participants expressed frustration with the US health care system, which requires a visit to a health care provider to obtain a prescription for seemingly low-risk treatments for common problems. There was little or no recognition of the impact of self-medication with antibiotics on antibiotic resistance. This group believed that the United States was unnecessarily expensive and regulatory compared with Latin American countries, where antibiotics are more easily available. The participants felt that it was not worth the expense or the hassle to obtain antibiotics through physician visits when other strategies, like purchasing antibiotics in tiendas and importing antibiotics from relatives, were available and perceived as safe.

These results are troubling from a public health standpoint with regards to both appropriate diagnosis and treatment, yet they are not unexpected. It would be expected that when patients are diagnosed and given a treatment for a condition, after the use of which they are better, they would consider that diagnosis to be correct and the treatment effective. It would be unrealistic to believe that patients would disregard previous experience in seeking care and treatment expectations every time they have a respiratory infection. ${ }^{13,17-19}$ Consequently, a desire for antibiotics previously used to treat respiratory infections is not a Latino-specific cultural phenomenon. However, the use of a variety of strategies, including acquiring antibiotics without a prescription from tiendas and pharmacies within the United States, does seem to be a cultural artifact of the loosely regulated sale of antibiotics in Latin America. ${ }^{20}$ This practice has appeared in cities such as New York, with longstanding Latino populations, and is present in cities like Charleston, which have seen the relatively recent influx of Latinos.

This study adds to the growing body of knowledge about the need to devise effective interventions to deal with the self-medication of antibiotics in the Latino community. ${ }^{19}$ Currently, there are no published results of interventions to combat this behavior, which has significant implications for the development of antibiotic resistance and public health. ${ }^{21}$ Education about any sort of intervention to promote the use of appropriate channels for acquiring antibiotics and to decrease inappropriate self-medication seems necessary, but a strategy of educating individuals about the differences between viruses and bacteria in the etiology of upper respiratory infections would probably not be effective. The participants base their desire for antibiotics not on the perceived etiology of the illness but on previously successful, or perceived successful, treatment for an infectious symptom complex. Thus, they are not looking to treat bacteria versus viruses but rather to alleviate symptoms. Furthermore, educational interventions for physicians will have to be done with care because most of the care seeking by this population is outside of the formal medical sector.

A multifactorial strategy focusing on the need to see a doctor before self-medicating with antibiotics is a logical step based on these findings. A message that focuses on the potential risks of self-medicating with antibiotics would seem to have an increased chance for success. Because of this unique cultural strategy of bypassing the formal health care system to obtain antibiotics, we have been working with the Centers for Disease Control and Prevention's Get Smart program, as well as the South Carolina Department for Health and Environmental Control, on the development of educational materials for the Latino patient community. The present data indicate that translating currently used pamphlets on antibiotic use into Spanish would miss the entire process of self-medication with an- 
tibiotics acquired without a prescription. New patient educational materials, termed "Solo con receta" ("Only with prescription"), were developed with the Centers for Disease Control and Prevention and are being disseminated as an adjunct to radio and television health programs and as public service messages in Spanish-language media.

Another strategy that may work would be to disseminate the messages via a promotore program. Promotores can act as a liaison to the Latino community and disseminate information about the appropriate management of disease and activities for prevention. These promotores can provide culturally sensitive educational materials to patients and can help the community to connect with the health system and overcome conflicts in health beliefs and communication.

One concern about conceptualizing an intervention for the Latino community is the assumption that people who are from a variety of countries in Central America, South America, and the Caribbean can all be lumped into a general rubric of Latino. The present results from a community that has seen a relatively recent surge of Latino immigration, primarily from Mexico, are consistent with those from a recent study using focus groups of Latinos in New York City, who were primarily from the Dominican Republic. ${ }^{19}$ This suggests that although cultural differences among Latino groups do exist and community structures may vary, this behavior of self-medication with antibiotics may be common to the general Latino community. One difference evident between the primarily Mexican participants in our study and the Dominicans in New York City was the reliance on the importation of antibiotics with Mexicans, whereas the availability of antibiotics without a prescription at bodegas in New York seems to be a more accepted strategy for acquiring treatment. As the Latino community grows in Charleston, more outlets for acquiring antibiotics without a prescription may appear.

This study has several limitations. First, this study only involved community members and did not include proprietors of tiendas or other suppliers of antibiotics without a prescription. Such suppliers may provide further insight into the strength of the cultural acceptance of this process, which is inconsistent with US health care regulations. The study did achieve saturation from community members who self-medicate with antibiotics. The owners of the tiendas are not known users of antibiotics with- out a prescription and the rationale for this study was to explore the motives and experiences of the users of unprescribed antibiotics. However, future studies focusing on drug suppliers can help to explain other aspects of how the phenomenon persists (eg, How do they get the antibiotics into the country?, Are they scrutinized by the Food and Drug Administration or law enforcement?, To whom do they decide to sell antibiotics without a prescription?). A second limitation is that the participants are all immigrants to the United States. Second generation Latinos may have different behaviors and attitudes toward self-medication with antibiotics.

\section{Conclusion}

Interventions are necessary to promote the appropriate use of antibiotics in the Latino community in the United States. Successful interventions need to move beyond simply translating interventions developed for the mainstream US population; rather, they need to be culturally sensitive to specific attitudes and behaviors found in the Latino population.

Special thanks to Tara Hogue for her help with the focus groups and Cindy Friedman, MD and the Get Smart initiative of the Centers for Disease Control and Prevention for their input on the focus group questions.

\section{References}

1. Neu HC. The crisis in antibiotic resistance. Science 1992;257:1064-73.

2. Cohen ML. Epidemiology of drug resistance: implications for a post-antimicrobial era. Science 1992; 257:1050-5.

3. Becker J, Drucker E, Enyong P, Marx P. Availability of injectable antibiotics in a town market in southwest Cameroon. Lancet Infect Dis 2002;2:325-6.

4. Hsueh P-R, Luh K-T. Antimicrobial resistance in Streptococcus pneumoniae, Taiwan. Emerg Infect Dis 2002;8:1487-91.

5. Dua V, Kunin CM, White LV. The use of antimicrobial drugs in Nagpur, India. A window on medical care in a developing country. Soc Sci Med 1994;38: 717-24.

6. Multicenter study on self-medication and self-prescription in six Latin American countries. Drug Utilization Research Group, Latin America. Clin Pharmacol Ther 1997;61:488-93.

7. Calva J. Antibiotic use in a periurban community in Mexico: a household and drugstore survey. Soc Sci Med 1996;42:1121-8.

8. Kleinman A, Eisenberg L, Good B. Culture, illness, and care: clinical lessons from anthropologic and 
cross-cultural research. Ann Intern Med 1978;88: 251-8.

9. Pachter LM. Culture and clinical care: folk illness beliefs and behaviors and their implications for health care delivery. JAMA 1994;271:690-4.

10. Fishman BM, Bobo L, Kosub K, Womeodu RJ. Cultural issues in serving minority populations: emphasis on Mexican Americans and African Americans. Am J Medical Sci 1993;306:160-6.

11. Mangione-Smith R, Elliott MN, Stivers T, McDonald L, Heritage J, McGlynn EA. Racial/ethnic variation in parent expectations for antibiotics: implications for public health campaigns. Pediatrics 2004;113:e385-94.

12. Corbett KK, Gonzales R, Leeman-Castillo BA, Flores E, Maselli J, Kafadar K. Appropriate antibiotic use: variation in knowledge and awareness by Hispanic ethnicity and language. Prev Med 2005;40: 162-9.

13. McKee MD, Mills L, Mainous AG III. Antibiotics for the treatment of upper respiratory infections in a diverse community. J Fam Pract 1999;48:993-6.

14. Mainous AG III, Cheng AY, Garr RC, Tilley BC, Everett CJ, McKee MD. Nonprescribed antimicrobial drugs in Latino Community. South Carolina. Emerg Infect Dis 2005;11:883-8.

15. US Census Bureau, US Department of Commerce Economics and Statistics Administration. The Hispanic population: census 2000 brief; 2001.

16. McCracken G. The long interview. In: Miller ML, Van Maanen J, Manning PK, editors. Qualitative research methods series. Newbury Park (CA): Sage Publications; 1988.

17. Dosh SA, Hickner JM, Mainous AG III, Ebell MH. Predictors of antibiotic prescribing for nonspecific upper respiratory infections, acute bronchitis, and acute sinusitis. An UPRNet study. Upper Peninsula Research Network. J Fam Pract 2000;49:407-14.

18. Mainous AG III, Zoorob RJ, Oler MJ, Haynes DM. Patient knowledge of upper respiratory infections: implications for antibiotic expectations and unnecessary utilization. J Fam Pract 1997;45:75-83.

19. Larson EL, Dilone J, Garcia M, Smolowitz J. Factors which influence Latino community members to self-prescribe antibiotics. Nurs Res 2006;55:94-102.
20. Larson E, Grullon-Figueroa L. Availability of antibiotics without prescription in New York City. J Urban Health 2004;81:498-504.

21. Cespedes A, Larson E. Knowledge, attitudes, and practices regarding antibiotic use among Latinos in the United States: review and recommendations. Am J Infect Control 2006;34:495-502.

\section{Appendix 1. Issues Discussed in Focus Groups Related to the Use of Antibiotics Obtained Without a Prescription (English translation)}

Have you ever used antibiotics that were not prescribed by a doctor or other health care professional?

Probe: Give a recent example tell your story.

Probe: Where did you learn to treat that illness with antibiotics? mother, doctor (here or there), pharmacist (here or there), friend.

Probe: Where did you get them?

(Ask about tiendas)

Probe on differences based on length of time in United States

Why did you self-medicate with antibiotics without seeing a doctor?

Probe on barriers: time, money, insurance, transportation, language, believe it to be unnecessary.

Would you treat your children the same way you treat yourself?

What is your attitude toward the use of antibiotics without a prescription?

Probe: Saves money, convenience, no risk in the use of the medications

What are the risks/bad things related to the inappropriate use of antibiotics?

Probe: If none, then would their beliefs change if the thought that antibiotics could cause them to get diarrhea, infections that can't be cured?

How should we teach people to use antibiotics appropriately? 